Review

\title{
Celiac Disease and Associated Extraintestinal Manifestations, with Special Reference to Neurological Disorders
}

Luis Rodrigo ${ }^{1,2, *}$

1. Emeritus Professor of Medicine, Oviedo University, Oviedo, Spain; E-Mail: Irodrigosaez@gmail.com

2. Honorary Chief of the Digestive System Service, Oviedo, Spain

* Correspondence: Luis Rodrigo; E-Mail: Irodrigosaez@gmail.com

Academic Editor: Bart Ellenbroek

Special Issue: Gluten-Related Neurological Disorders

OBM Neurobiology Received: July 13, 2020

2020, volume 4, issue 4

Accepted: October 19, 2020

doi:10.21926/obm.neurobiol.2004074

Published: October 30, 2020

\begin{abstract}
Celiac disease $(C D)$, also known as gluten-sensitive enteropathy and nontropical sprue, is one of the most important entities of the wide spectrum of gluten-related disorders (GRDs). It is well known that neurological manifestations can be present either at the onset of $C D$, or appear during the development of the pathology, and the neurologic findings can show a profound difference. Clinical features show significant variation, ranging from typical manifestations of gastrointestinal involvement to several extraintestinal diseases. The most frequent neurologic signs reported are headaches, epileptic seizures, and migraines. Polyneuritis and the cerebellar ataxias have shown the most frequent association in adults. Headache either in the form of migraine or in nonspecific form represents one of the main clinical presentations in $C D$. This work aims to provide a narrative review of the current state of $C D$, highlighting the main extraintestinal disorders associated with $C D$. Complete compliance to a gluten-free diet (GFD) seems to improve the neurological symptoms even though the underlying pathogenic relationship between $C D$ and the neurologic system remains poorly understood.
\end{abstract}

\section{Keywords}

Celiac disease; non-celiac gluten sensitivity; gluten-free diet; extraintestinal manifestation; associated neurological disorders 


\section{Introduction}

Celiac Disease (CD) is a chronic autoimmune inflammatory enteropathy that affects genetically predisposed individuals and is characterized by the presence of permanent gluten intolerance. It has a worldwide distribution [1]. It affects mainly the upper portion of the small intestine. The most common digestive clinical manifestations are the presence of slow and heavy digestion, accompanied by frequent episodes of abdominal bloating that are aggravated by the intake of certain foods. It is usually accompanied by diarrhea and moderate weight loss [2].

\section{Risk Groups}

First-degree relatives have been confirmed by duodenal biopsy as having an average prevalence of $C D$ of around $12 \%$. The second-degree relatives may also be affected at a higher degree than the general population, as is clearly shown by family studies, which prove extremely useful whenever a patient is diagnosed with celiac disease $[3,4]$.

$C D$ is present at an even higher frequency among people with Down syndrome and also occurs in individuals with other genetic alterations, such as Turner syndrome (TS) and Williams syndromes (WS), immunoglobulin A (IgA) deficiency, and a wide variety of autoimmune processes (Table 1).

Table 1 Risk groups and CD-associated diseases.

\begin{tabular}{ll}
\hline A & First-degree relatives \\
B & Genetic diseases: Down, Turner, Williams syndromes, \\
& etc. \\
C & Selective IgA deficiency \\
& Endocrinological diseases \\
D & -Insulin-dependent diabetes mellitus (type 1) \\
& -Thyroid diseases (hyperthyroidism, hypothyroidism) \\
& Neurological diseases \\
& -Epilepsy with occipital calcifications (Gobbi \\
E & syndrome [CEC syndrome]) \\
& -Cerebellar ataxia \\
& Rheumatic diseases \\
& -Rheumatoid arthritis (RA) \\
& -Sjögren's syndrome (keratoconjunctivitis sicca) \\
F & Skin diseases \\
& -Psoriasis \\
& -Vitiligo \\
& -Alopecia areata (AA)
\end{tabular}




\begin{tabular}{|c|c|}
\hline & Heart diseases \\
\hline \multirow[t]{3}{*}{$\mathrm{H}$} & -Dilated cardiomyopathy \\
\hline & -Recurrent pericarditis \\
\hline & Liver diseases \\
\hline \multirow[t]{3}{*}{ I } & -Autoimmune hepatitis (AlH) \\
\hline & -Primary biliary cholangitis (PBC) \\
\hline & Kidney disease \\
\hline \multirow[t]{3}{*}{ J } & -IgA nephropathy \\
\hline & -Nephrotic syndrome \\
\hline & Collagenosis \\
\hline \multirow[t]{5}{*}{ K } & -Dermatomyositis \\
\hline & -Systemic lupus erythematosus (SLE) \\
\hline & Digestive diseases \\
\hline & -Gastroesophageal reflux disease (GERD) \\
\hline & -Inflammatory bowel disease (IBD) \\
\hline \multirow[t]{4}{*}{$\mathrm{L}$} & -Collagenous colitis, lymphocytic gastritis \\
\hline & -Functional dyspepsia \\
\hline & -Irritable bowel syndrome (IBS) \\
\hline & -Recurrent acute pancreatitis \\
\hline
\end{tabular}

A relatively high proportion of patients [3-8\%] with type 1 diabetes mellitus (T1DM) present associated disorders of $C D$. The treatment of diagnosed CD with a gluten-free diet (GFD) in juvenile diabetic patients may not completely alleviate the condition but may greatly facilitate better metabolic control, help prevent certain complications, improve patients' quality of life, and notably reduce the need for insulin [5].

On average, approximately $5 \%$ of people with hyperfunction or hypofunction in thyroid diseases also present CD; the prevalence reaches 25\% among those who test positive for antithyroid antibodies. As in people with diabetes, in most cases, establishing a GFD helps correct the associated thyroid dysfunction, reduces the dose of hormonal therapy required, and sometimes even enables suspension of the therapy [6].

The more common gastrointestinal manifestations of $C D$ include recurrent abdominal distension generally associated with pain, prolonged and difficult digestions, alterations of feces evacuation (diarrhea or constipation), and weight loss. CD can present in association with a huge diversity of diseases affecting various organs. Dermatitis herpetiformis [DH] is an example of gluten-induced skin disease, with associated extradigestive manifestations (EDMs) [7], though there are other gluten-related diseases also, such as those of the liver, nervous system, bone, and those involved in rheumatological, cardiac, renal, collagenous, and other processes [8-10] (Table 2). 
Table 2 Extradigestive manifestations of celiac disease.

$>$ Delayed growth and development

$>$ Osteopenia, osteoporosis, arthritis, pathological fractures

$>$ Generalized bone and muscle pain

Iron deficiency anemia, leukopenia, thrombopenia, hypoprothrombinemia (hemorrhagia)

Ankle edema (hypoalbuminemia), muscle cramps, tetany (hypocalcemia)

> Reproductive disorders (late menarche, amenorrhea, hypermenorrhea, early menopause, miscarriage, infertility, impotence, increased neonatal morbidity)

Depression, anxiety, irritability

Sleep disorders: insomnia, hypersomnia

$>$ Acute, persistent, and unmotivated asthenia

$>$ Fibromyalgia

$>$ Hyposplenism

$>$ Reactive hepatitis (idiopathic hypertransaminasemia)

$>$ Recurrent aphthous stomatitis

$>$ Hypoplasia of dental enamel

Dermatitis herpetiformis (DH)

Several authors have previously discussed the prevalence of autoimmune processes in CD. Ventura et al. [16] studied it in the context of the timing of exposure to gluten, showing that when the disease is diagnosed late in childhood or during adolescence, the estimated relative risk of presenting an associated autoimmune process during a person's lifetime is about seven times higher than if diagnosed early in childhood.

In order to diagnose $\mathrm{CD}$ accurately, apart from considering the clinical data, the serological and genetic markers must be assessed, duodenal biopsies must be performed, and in doubtful cases, a GFD should be tried and the patient's clinical response should be assessed.

\section{Serological Markers}

Several serological markers are currently available, such as anti-gliadin antibodies (AGAs), antiendomysial antibodies (anti-EMAs), and those directed against type 2 tissue transglutaminase (tTG) (an enzyme present in various organs; particularly responsible for deamidating gliadin in the small intestine). IgA class antibodies have proven to be the most sensitive markers in the diagnosis of CD.

AGAs have the lowest diagnostic sensitivity and specificity (around 50\%) of all these markers, except in children aged under 18 months. Thus, AGAs are no longer used for diagnosis and followup of CD in children older than 18 months, adolescents, or adults of any age. 
tTG is the main antigen against which anti-EMAs react, although other minor antigens may also be located in the endomysium. Possibly due to this reason, the results of the determinations of the anti-EMAs and the tTG show no concordance. In general, it seems that anti-EMAs are more specific, and anti-transglutaminase antibodies are more sensitive, especially when a recombinant human antigen is used. In general, these markers have high sensitivity and specificity (close to $90 \%$ ) in the presence of marked atrophy of the intestinal villi. However, they are markedly less sensitive (by $40-50 \%$ ) in cases with mild villous atrophy or minimal changes. Conversely, these markers can also be positive in cases with normal mucosa or with minimal changes [17, 18].

It is noteworthy that the various reagents commercially available for use in ELISA or immunofluorimetric techniques have different sensitivities and specificities, so laboratories should specify the type of technique they use and their recommended reference values [19].

Selective IgA deficiency is ten times more frequent in celiacs (1/40) than in the general population (1/400). Hence in CD cases, IgG class antibodies are suggested for a definitive diagnosis.

It is mention-worthy that some celiac patients have negative serology, especially those with a normal duodenal biopsy or with minimal changes, in whom the diagnosis of CD is difficult, or at least debatable. In general, the tTG works worse in everyday clinical milieu than in research studies, most of which are carried out in children [6].

\section{Genetic Markers}

Human leukocyte antigen- class II (HLA-DQ2) heterodimer gene is present in $90 \%$ of cases of patients with CD, and HLA-DQ8 in $5-10 \%$ of patients. In southern European countries, up to $5 \%$ of patients can be DQ2- and DQ8-negative even though one of the alleles responsible for these two specificities is usually present. These susceptibility markers are widespread in about one-third ([around 30-40\%) of the general population, so the high negative predictive value of these alleles makes their absence remarkable [20]. The presence of these markers is necessary but not sufficient for the disease to develop and is important when undertaking family studies and/or recommending the adoption of a GFD in patients whose diagnostic tests show inconclusive results.

Other class I HLA system (HLA-I) genes, such as MHC class I chain-related gene A (MICA) and MHC class I chain-related gene $B(M I C B)$ are associated with a variety of autoimmune diseases, including CD [21].

Monozygotic twins show a high (around 75\%) concordance for presenting CD throughout their lives, while dizygotic twins show no difference in their relative risk compared with other firstdegree relatives, with an average prevalence of around 10\% [22].

\section{Duodenal Histological Markers}

Taking multiple biopsies of the small intestine continues to be considered a "gold standard", the most important test for confirming a diagnosis of the CD. As such, it should be performed whenever there is a serious clinical suspicion, regardless of the results from the serological markers. Histological confirmation of the diagnosis is very important since the patient requires special dietary treatment for the rest of their life to manage the disease. The change in diet is significant, often expensive, and entails serious social limitations.

Duodenal biopsies must be obtained by endoscopy since endoscopy enables a conspicuous view of the stomach and duodenum and facilitates multiple biopsies in a short time. It is advisable 
to obtain 4-6 biopsy samples from different parts of the distal duodenum so that the pathologist has sufficient material because the disease, although diffuse, can sometimes be accompanied by patchy involvement, and the small size of the biopsies makes the samples difficult to orientate appropriately before processing.

The indications for taking duodenal biopsies during an upper digestive endoscopy are varied and include not only patients with positive CD serology whose diagnosis requires confirmation, but also all patients with chronic or recurrent diarrhea, iron-deficiency anemia of unknown origin, those who are refractory to oral replacement therapy, or who have prolonged osteoporosis, patients with continuous maldigestion or relapsing abdominal pain, sustained weight loss, etc. regardless of the nature of their serology.

The identification of macroscopic signs such as the scalloping of the mucous folds, their decrease in size, or the presence of a "mosaic pattern" is suggestive of villous atrophy and underlines the need for biopsy by an expert endoscopist, although their absence does not in any way rule out $\mathrm{CD}$.

The spectrum of pathological changes in CD is very broad and is not limited to the presence of intestinal villous atrophy, as was thought hitherto. Instead, the spectrum covers practically normal biopsies (Stage 0), those that only show an increase in the intraepithelial lymphocytic infiltrate (Stage 1), those with compensatory crypt hyperplasia, accompanied by chronic inflammation in the lamina propria (Stage 2), and those with atrophy of the intestinal villi (Stage 3). Stage 3 is subdivided into three categories according to the nature of intensity of the atrophy: mild (3a), moderate (3b), or intense (3c) (Table 3).

Table 3 Marsh classification of duodenal lesions in celiac disease.

Stage Histological characteristics

1: Infiltrative

2: Hyperplastic

3: Atrophic

$3 a$

$3 b$

$3 c$

Crypt hyperplasia
Increased frequency of intraepithelial lymphocytes (IELs) (> 25 per 100 epithelial cells)

Chronic inflammatory infiltrate in the submucosa

Mild or partial villous atrophy

Subtotal or moderate villous atrophy

Total or complete villous atrophy

This anatomopathological classification of duodenal biopsies was first described by Dr. Marsh in 1992 [23]. Since then, it has been widely used throughout the world, clearly demonstrating its great diagnostic utility and the broad spectrum of histological alterations existing in $C D$, ranging from normal or minimally changed mucosa to the presence of atrophy of the intestinal villi.

The histopathological findings described are characteristic of, but not specific to, CD. This allows a presumptive diagnosis to be made since villous atrophy can occur in other intestinal processes of various etiologies that are not related to gluten intolerance (Table 4). 
Table 4 Other causes of non-celiac disease villous atrophy.

Giardiasis
Variable common immunodeficiency
Whipple's disease
Post-radiation enteritis
Enteropathy associated with HIV
Intestinal lymphoma
Zollinger-Ellison syndrome
Autoimmune enteropathy
Crohn's disease
Other food allergies (milk, soy, chicken, tuna)

There is a positive correlation between the positivity of anti-tTG antibodies and the degree of villous atrophy present in the duodenal mucosa, whereas greater atrophy is associated with greater antibody positivity, as occurs in the classic forms that predominate in children. Conversely, when the duodenal biopsy exhibits a low degree of atrophy or shows minimal changes, a lower level of antibody positivity is witnessed. There is no difference in the diagnosis of CD between adults and children. However, studies [24, 25] showed that in children with high titers of anti-TGt [>100], performing duodenal biopsies to confirm the diagnosis is not necessary (Figure 1).

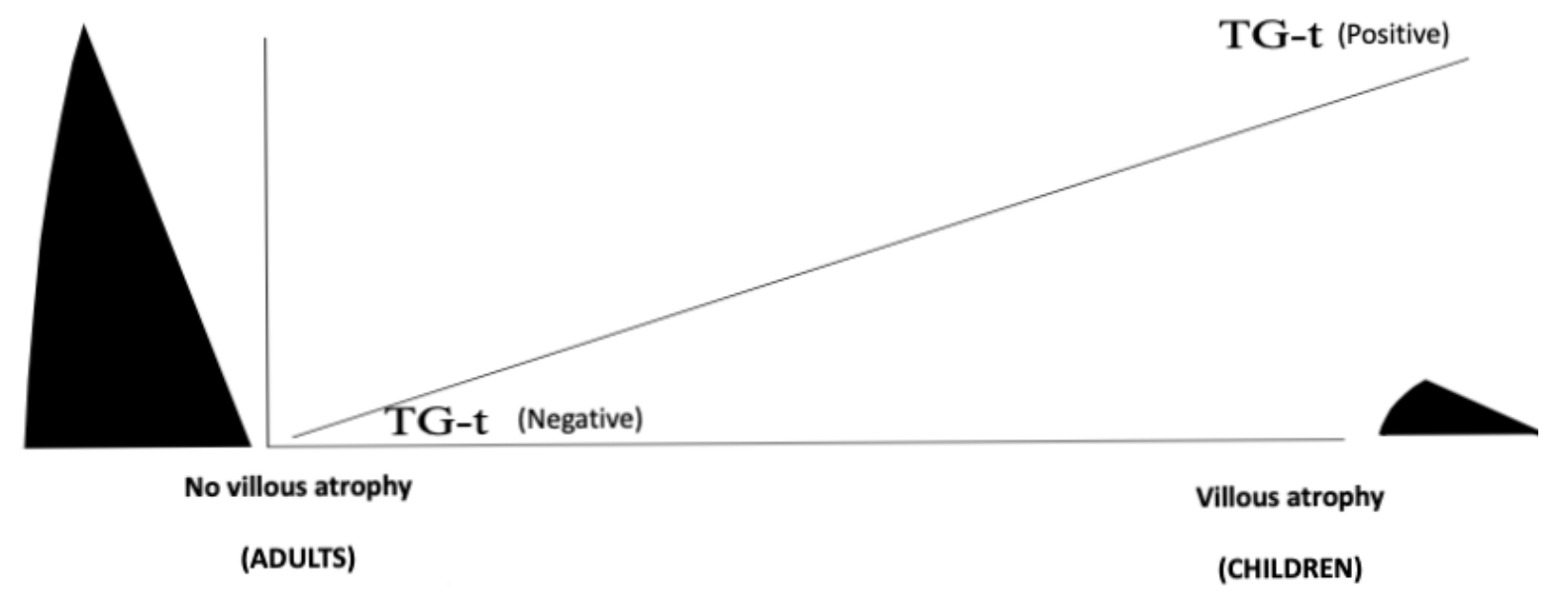

Figure 1 Relationship between positive serology and villous atrophy.

The diagnosis of CD is also confirmed when there is a favorable response to the GFD, which is assessed after an average period of six months.

\section{Diagnostic Criteria}

It is highlighted that no single test alone can conclusively diagnose CD or confirm the possibility of the presence of $C D$ in an individual.

This precise combination of clinical, analytical, and duodenal biopsy data and the response to the GFD makes diagnosis easier to reach. 
The routine application of the Marsh classification to study duodenal biopsies is very helpful for identifying hidden, doubtful cases. The practice should be adopted by all pathology services since it not only facilitates the use of a common language but also leads to the better classification of findings and an appreciation that the presence of villus atrophy is not essential to confirm the diagnosis, since, as the clinical one, the morphological spectrum of duodenal lesions is very wide.

When doubtful cases of $C D$ arise or a diagnosis proves difficult, various strategies may be adopted in accordance with the preferences of the patients and their families. In such circumstance, we highlight the following strategies: periodic repeated serology and analytics (of little use); performing a new endoscopy with biopsy after a few weeks, with or without gluten overload (less accepted); performing a trial treatment of a GFD for six months; observing the clinical response; and the analytical and serological changes (the most highly recommended). We include them in the diagnostic algorithm illustrated below (Figure 2).

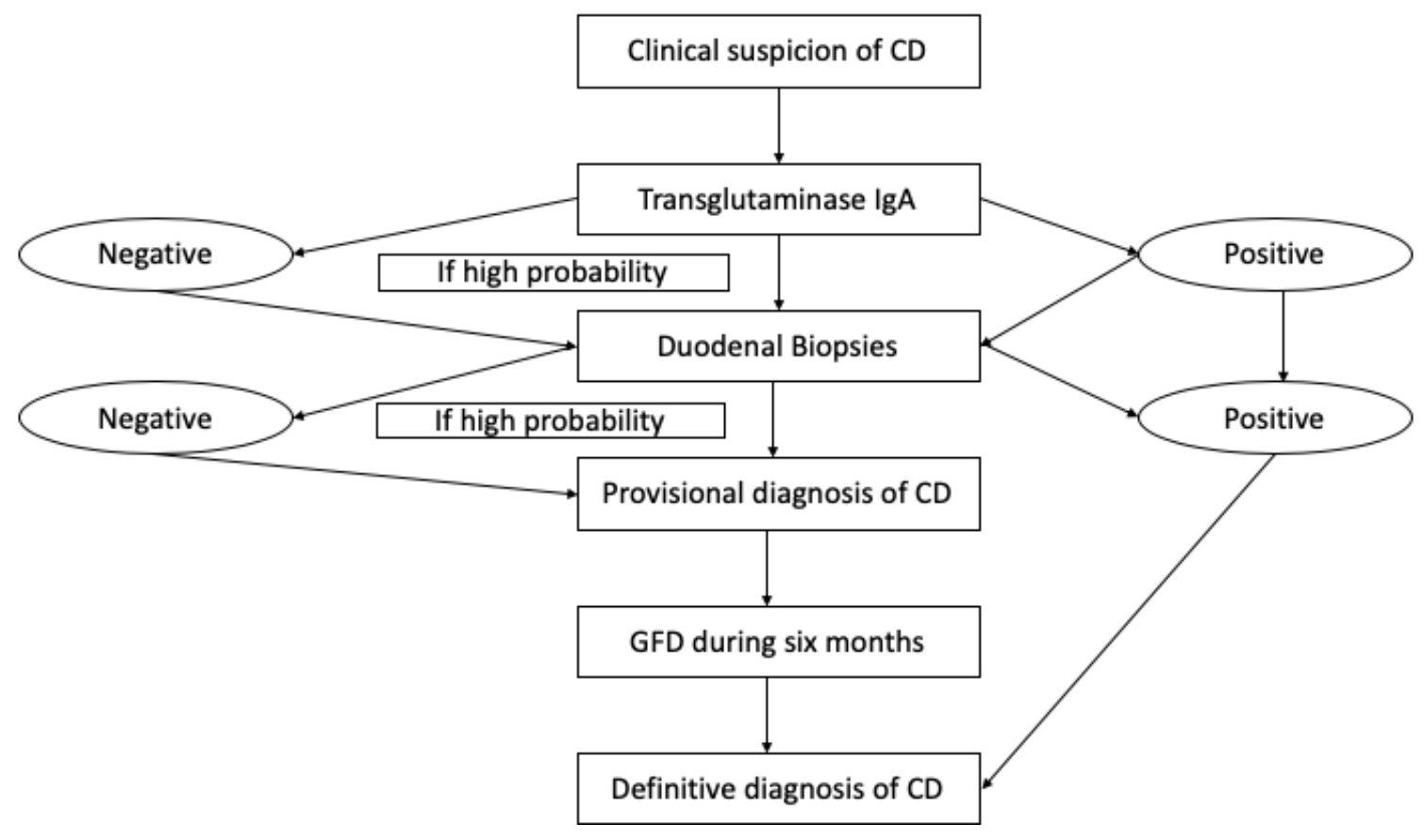

Figure 2 Relationship between positive serology and villous atrophy.

\section{Associated Diseases}

In recent decades, various extraintestinal manifestations, including iron deficiency, osteoporosis, T1DM, dermatitis herpetiformis, Hashimoto's autoimmune hypothyroidism, and several other neurological processes have been found to be associated with CD. Recent studies have confirmed that celiac individuals are 3-10 times more frequently associated with autoimmune diseases than the general population [26]. Besides, a genetic predisposition has been frequently observed since the celiacs often have genes known to favor the presence of two or more associated diseases [27]. The delayed diagnosis of CD, especially in adults, and the prolonged exposure to gluten could also favor the appearance of associated diseases, although this hypothesis remains unconfirmed [11]. It can be concluded that $C D$, like other autoimmune disorders, presents several associated immune complications that have only recently been discovered. 
Finally, it is worth recalling that non-celiac gluten sensitivity (NCGS), which is considered a minor variant of $C D$ because it lacks the serological, genetic, and histological markers characteristic of $C D$, is frequently accompanied by the same extra-digestive manifestations as a $C D$. As is the case for CD, patients who present NCGS benefit from the dietary effects of removing gluten from their diet, thereby experiencing a clear improvement in their evolution with a clinically significant reduction in associated clinical discomfort [28].

Some of the clinical manifestations frequently associated with CD are reviewed below.

\section{Iron Deficiency Anemia (IDA)}

Iron-deficiency anemia accompanied by $\mathrm{CD}$ at diagnosis and during its evolution occurs in varying proportions, with an average frequency of $5-15 \%$ in cases of cases [29]. In the same pattern, studying patients with recurrent or prolonged episodes of anemia often predisposes them to an underlying $C D$ that, in most cases, has not previously been diagnosed [30].

The most common cause of occurrence of anemia is the prolonged decrease in intestinal absorption of iron, as a consequence of inflammatory changes produced by gluten in the duodenum of celiac patients. This alteration is evidenced by the lack of response to treatment with orally administered iron, which is re-established upon treatment with a GFD. Other associated factors may also help maintain the anemia, which, in these cases, can have a multifactorial origin [31].

\section{Osteoporosis}

Metabolic bone disease (with osteoporosis being the commonest paradigm) is a complication frequently associated with the clinical evolution of $C D$, especially at the time of its diagnosis in children and adults.

It is estimated that, at the time of $C D$ diagnosis, one-third of pediatric patients have osteoporosis, one third have osteopenia, and the rest display no detectable bone abnormalities.

The reported [32] prevalence of osteoporosis in adult CD patients is twice that in the unaffected control population of the same age and sex. Low bone mineral density (BMD) has been documented in patients with classic $C D$, those with mild digestive manifestations, and even in asymptomatic celiac patients. Due to this reason, no clinical correlation between BMD and CD could be established yet. Detailed studies are suggested to evaluate osteoporosis in most adult celiacs in order to detect the condition and treat it early.

The etiology of osteoporosis in CD is multifactorial and is influenced by various components, including genetic, environmental, hormonal, and associated chronic inflammatory processes [33].

\section{Dermatitis Herpetiformis}

Dermatitis herpetiformis (DH) was first described in 1885 by the French dermatologist Louis A. Duhring. The characteristic cutaneous lesions appear in the form of erythematous papules associated with vesicles containing a slightly yellowish liquid, distributed symmetrically over the entire body surface, located especially in the extensor areas of the arms, legs, hands, as well as in the intertriginous areas, such as the buttocks, fingers, and toes. However, mucosal involvement is 
rare. The lesions can appear at any age and are accompanied by intense itching, aggravated by heat and friction, and very troublesome at night as that they disturb sleep [34-36].

Most patients with $\mathrm{DH}$ present associated digestive discomfort that is neither continuous nor conspicuous. In general, males are more frequently affected than females (1.5-2:1). In skin biopsies close to the affected areas, the most characteristic findings consist of the presence of granular IgA deposits, located in the dermal papillae, as well as in the basal membrane, as demonstrated by specific immunofluorescence. They are presented as immunological responses against class 3 anti-tTG antibodies, which are characteristic of celiac patients with associated DH.

DH has been found commonly associated with HLA-DQ2 and/or HLA-DQ8, the genetic markers of susceptibility [37, 38].

The most effective treatment for DH is the GFD, which must be strictly and continuously adhered to throughout life. The skin lesions disappear completely a few weeks after starting the GFD. These lesions occur in $25 \%$ of celiac patients and are specific to gluten intolerance, so their presence informs and confirms the suspected diagnosis in doubtful cases. Since duodenal biopsies, although suggestive, are not always definitive in these cases of $C D$, and in many cases, inflammatory changes are more accurately diagnosed as in NCGS [39]. The epidemiological distribution of both $\mathrm{DH}$ and $\mathrm{CD}$ is worldwide, with more prevalence in humid and warm countries.

\section{Type 1 Diabetes Mellitus (T1DM)}

The close association between CD and T1DM has been known for more than 50 years, and, generally, this marked tendency has led to two manifestations found to co-occur in $5-15 \%$ of CD patients [40]. This relationship may be because both diseases partially share the same genetic basis, and the DQ2/DR3 haplotypes are frequently present in the CD/T1DM patients [41], although both diseases are polygenic and have a greater susceptibility in several common loci, distributed over different chromosomes.

In more than $90 \%$ of $C D$ patients who also have T1DM (child and adolescent forms), the diabetes is diagnosed first, followed by the diagnosis of $C D$ after months or more often years later.

Patients with T1DM and associated CD who adopt a GFD showed improved general health and markedly better digestion. Improved metabolic control is demonstrated by decreasing the previously administered insulin dose to more stable and sustained doses and by better blood glucose levels. Hypoglycemic episodes also become less frequent and less severe, and patients' diabetes becomes more stable, and its control is simpler. Although it is not possible to control it completely without insulin by lowering their daily needs, it significantly improves the quality of life of diabetics [42].

It is recommended to screen children and adolescent patients systematically with T1DM for CD at least annually for a minimum of five years, starting from the time of the first diagnosis of diabetes [43]. Serological tests are very effective at identifying CD. In T1DM patients with positive serology for $C D$, the diagnosis must be completed with the determination of genetic markers (DQ2 and DQ8) and duodenal biopsies to complete the diagnosis [44].

\section{Autoimmune Hypothyroidism}

$C D$ is frequently associated with thyroid processes, in the form of hyperthyroidism and autoimmune hypothyroidism. The latter is more frequent, with an average incidence of $2-7 \%$, but 
can reach $25 \%$ during its evolution [45]. Thyroid dysfunction was found to be at least thrice as common in celiac adults as in controls of the same age and sex [46].

In a recent study of 74 adult patients with CD, carried out in India, at least one associated endocrine disease, either clinically evident or subclinical, was found in 31 cases $(41.9 \%)$, and multiple associated endocrine processes occurred in nine cases (12.2\%). Of these, the most frequent were T1DM and autoimmune thyroid disease. Furthermore, eight patients (10.8\%) had functional hypopituitarism, and 7 (12.9\%) out of 54 patients had hypogonadotropic hypogonadism. In this review, it is concluded that CD is associated with a high frequency of several clinically manifested endocrinopathies, including dysfunction of the pituitary-gonadal axis and other subclinical ones. Establishing a GFD early on can help reverse various subclinical endocrinopathies and improve the control of the manifest conditions [47].

Similarly, various authors suggested that patients with autoimmune thyroiditis who do not improve with hormone replacement therapy, or who present general nonspecific symptoms despite strictly following their treatment, should be studied to determine the presence of other associated autoimmune disorders including $C D$ to avoid diagnostic delay, thereby helping in better recovery [48].

\section{Associated Neurological Processes}

There are a great variety of associated neurological processes, including polyneuritis and mononeuritis; ataxias; seizure disorders; impaired cognitive functions; migraines; and several other neurological disorders (in decreasing order of frequency). These are shown in Table 5.

Table 5 Neurological manifestations associated with CD and/or NCGS.

\begin{tabular}{lll}
\hline $\begin{array}{l}\text { Manifestation } \\
\text { Peripheral neuropathy }\end{array}$ & Prevalence (\%) & Author, Journal, and Year \\
Headaches & 49 & Cicarelli G et al., Neurol Sci, 2003 \\
Depression/Anxiety & 34 & Cicarelli G et al., Neurol Sci, 2003 \\
Cerebellar ataxia & 5.4 & $\begin{array}{l}\text { Pynnonen PA et al., } \\
\text { Psychosomatics, } 2004\end{array}$ \\
Migraines & 4.4 & Zelnik N et al., Pediatrics, 2004 \\
Epilepsy & $3.3-3.5$ & Cicarelli G et al., Neurol Sci, 2003 \\
\hline
\end{tabular}

Source: Green PH et al., Cell Mol Life Sci, 2005

\section{Polyneuritis and Mononeuritis}

The sensitive polyneuritis is the most frequent neurological process associated with $C D$, appearing in up to $50 \%$ of adult celiacs at some point during the evolution of polyneuritis [49]. Sometimes neuropathy may occur before the CD diagnosis and so should be suspected, especially if the neuritis is symmetric, predominantly distal, and predominantly sensitive [50]. 
Other forms are purely motor neuropathies such as multiple mononeuritis, Guillain-Barré syndrome, and autonomic neuropathy, which sometimes respond to treatment with a GFD. However, other patients continue to exhibit the same symptoms or even show progression after the withdrawal of gluten from the diet, in which case, the administration of intravenous immunoglobulins may prove beneficial [51].

\section{Cerebellar Ataxia}

Cerebellar ataxia is characterized by the appearance of gait disorders with incoordination, loss of balance, and associated dysarthria. It can appear in both CD and non-CD diagnosed patients. In some cases, only elevated serum AGAs are detected, and not all patients present with duodenal involvement that is compatible with $C D$, and so fit better within the NCGS framework.

A recent systematic review of 16 studies about the association between CD and at least one neurological disorder, published between January 2007 and September 2018, found associated neurological impairments in 13 studies, with more than $39 \%$ of the patients affected, on average. The review also noted that nine studies reported the prevalence of gluten ataxia in $0-6 \%$ among their cases. Adherence to a GFD seemed to improve symptoms in patients with neuropathy and ataxia. Medical professionals who treat these patients, including general practitioners, pediatricians, interns, and gastroenterologists, as well as neurologists who study patients with CD who have associated neuropathies of unknown origin, must be aware of, and actively check for this relationship between the processes, since switching to a GFD, even in suspect cases, such as those related to NCGS, can be highly beneficial for these patients. [52].

In a prospective study conducted by M. Hadjivassiliou in a gastroenterology center in Sheffield, UK, on 100 consecutive patients with a recent diagnosis of CD based on the findings of gastroscopy and duodenal biopsies, neurological deficits were found frequent, and $40 \%$ of the patients showed circulating antibodies against the transglutaminase-6 (TG6) idiotype. These patients exhibited significantly more atrophy of the subcortical regions, evaluated by MRI than by TG6-negative patients. This finding established a relationship between the autoimmunity against TG6 and brain atrophy in these patients. Those with altered pathology, as revealed by spectroscopic MRI of the cerebellum, showed improvements after one year on a GFD compared with baseline individuals [53].

A possible explanation involves the primary immune response to different potential autoantigens [transglutaminases] that produce different clinical manifestations, depending on the tTG subtype. Thus, in the case of isolated CD, the auto-antigen is TG2, in DH it is TG3, and in neurological manifestations, it is TG6, as confirmed in many patients with cerebellar ataxia [54-56].

Another systematic review of the neuropathological findings from 98 eligible patients with CD showed that those with gluten ataxia could also exhibit one or more additional patterns, including loss of Purkinje cells, cerebral atrophy, gliosis, associated astrocytosis, and the presence of a diffuse lymphocytic infiltrate with a predominantly perivascular aggregate. In patients with longfiber neuropathy, biopsies revealed the presence of axonopathy of myelinated fibers as well as focal and infiltrating perivascular inflammatory cells. Similar inflammatory infiltrates were found in the muscles of patients with myopathies and in the brains of patients with encephalopathy and epilepsy. These findings suggest that these lesions feature some immunopathogenic alterations. 
Further studies may help better identify the targets on which the inflammatory infiltrates, which are so characteristic of such lesion, act [57].

Although gluten ataxia is the best-known gluten-related neurological manifestation, other motor disorders may also be associated with gluten intolerance or sensitivity that can benefit from the adoption of a GFD by these patients. These have been reviewed recently [58].

\section{Seizures}

$C D$ is more frequently associated with several variants of childhood epilepsy than with adult epilepsy. The efficacy of a GFD in these cases has not yet been established. Some studies have shown a better control of childhood seizures, with a consequent reduction in the drugs administered to control them [59].

In Italy, in the 1970s, a childhood CD syndrome, Gobbi syndrome, with cerebral calcifications located at the occipital level came to light. The calcifications were usually bilateral and generally consisted of benign, rare calcified angiomas and fibrous veins. [60].

\section{Impaired Cognitive Function}

Cases of dementia associated with CD may occur. In most cases, following a GFD does not result in any improvement in the underlying neurological disorder. However, dementia in some patients is stabilized after removing gluten from their diet. Pathologic studies usually reveal unspecific gliosis in such cases [61, 62].

\section{Migraines}

The occurrence of migraines has been associated with several digestive processes, such as CD, Helicobacter pylori infection, and some functional disorders such as irritable bowel syndrome. Migraines may also be related to the activation of a variety of inflammatory mediators such as IL-6, IL-8, and TNF- $\alpha$. Several studies have found a higher prevalence (21-28\%) of migraines in CD patients than in the control population [63-65]. Some studies $[66,67]$ suggested that adopting a GFD might be beneficial because it reduces the frequency and intensity of migraine episodes

\section{Conclusions}

A possible relationship between other neurological diseases and CD has been described, in the form of rare cases and in short case series of patients. More studies in large series are suggested for comparisons between patients and control cases, or in the form of systematic reviews, in order to ascertain if these disorders are directly related to $C D$ or simply a coincidence, and thereby conclusively confirm or discount their relationship.

$C D$ may be associated with a range of neurological manifestations right from confirmed diagnosis to its evolution. Its presence should be checked in all neurological conditions where the cause cannot be identified and in cases of NCGS [68]. In cases where there is uncertainty but a strong suspicion of a possible association, it is better to adopt a GFD, and maintain it for at least one year, so that its efficacy may be assessed [69]. 
Overall, this review will enlighten the researchers and clinicians on the rapidly evolving spectrum of diseases associated with $C D$ and help suggest suitable interventions to reduce rising mortalities across the planet.

\section{Author Contributions}

Dr. Luis Rodrigo did all work.

\section{Competing Interests}

The author has declared that no competing interests exist.

\section{References}

1. McAllister BP, Williams E, Clarke K. A comprehensive review of celiac disease/gluten-sensitive enteropathies. Clin Rev Allergy Immunol. 2019; 57: 226-243.

2. Green PH, Krishnareddy S, Lebwohl B. Clinical manifestations of celiac disease. Dig Dis. 2015; 33: 137-140.

3. Freeman HJ. Celiac disease: A disorder emerging from antiquity, its evolving classification and risk, and potential new treatment paradigms. Gut Liver. 2015; 9: 28-37

4. Barker JM, Liu E. Celiac disease: Pathophysiology, clinical manifestations and associated autoimmune conditions. Adv Pediatr. 2008; 55: 349-365

5. Goodwin G. Type 1 diabetes mellitus and celiac disease: Distinct autoimmune disorders that share common pathogenic mechanisms. Horm Res Paediatr. 2019; 92: 285-292.

6. Sun $X$, Lu L, Yang R, Li Y, Shan L, Wang Y. Increased incidence of thyroid disease in patients with celiac disease: A Systematic review and meta-analysis. PLoS One. 2016; 11: e0168708.

7. Reunala T, Salmi TT, Hervonen K, Kaukinen K, Collin P. Dermatitis herpetiformis: A common extraintestinal manifestation of coeliac disease. Nutrients. 2018; 10: 602.

8. Nardecchia S, Auricchio R, Discepolo V, Troncone R. Extra-intestinal manifestations of coeliac disease in children: Clinical features and mechanisms. Front Pediatr. 2019; 7: 56.

9. Rodrigo L. Celiac disease. World J Gastroenterol. 2006; 12: 6585-6693.

10. Losurdo G, Principi M, lannone A, Amoruso A, lerardi E, Di Leo A, et al. Extra-intestinal manifestations of non-celiac gluten sensitivity: An expanding paradigm. World J Gastroenterol. 2018; 24: 1521-1530.

11. Ventura A, Magazu G, Gerarducci T, Greco L. Coeliac disease and the risk of autoimmune disorders. Gut. 2002; 51: 897.

12. Lewis NR, Scott BB. Systematic review: The use of serology to exclude or diagnose coeliac disease (a comparison of the endomysial and tissue transglutaminase antibody tests). Aliment Pharmacol Ther. 2006; 24: 47-54.

13. Abrams JA, Brar P, Diamond B, Rotterdam $H$, Green PH. Utility in clinical practice of immunoglobulin an anti-tissue transglutaminase antibody for the diagnosis of celiac disease. Clin Gastroenterol Hepatol. 2006; 4: 726-730.

14. Fernández $E$, Riestra $S$, Rodrigo L, Blanco $C$, López-Vázquez $A$, Fuentes $D$, et al. Comparison of six human anti-transglutaminase ELISA-tests in the diagnosis of celiac disease in the Saharawi population. World J Gastroenterol. 2005; 11: 3762-3766. 
15. Dickey W, Hughes DF, McMillan SA. Reliance on serum endomysial antibody testing underestimates the true prevalence of celiac disease by one fifth. Scand J Gastroenterol. 2000; 35: 181-183.

16. Kaukinen K, Partanen J, Mäkki M, Collin P. HLA-DQ typing in the diagnosis of celiac disease. Am J Gastroenterol. 2002; 97: 695-699.

17. López-Vázquez A, Rodrigo L, Fuentes D, Riestra S, Bousoño C, García-Fernández S, et al. MHC class I chain related gene $A$ (MICA) modulates the development of coeliac disease in patients with the high-risk heterodimer DQA1*0501/DQB1*0201. Gut. 2002; 50: 336-340.

18. Hogberg L, Falth-Magnusson K, Grodzinsky E, Stenhammar L. Familial prevalence of coeliac disease: A twenty-year follow-up study. Scand J Gastroenterol. 2003; 38: 61-65.

19. Marsh MN. Gluten major histocompatibility complex, and the small intestine: A molecular and immunobiologic approach to the spectrum of gluten sensitivity ("celiac sprue"). Gastroenterology. 1992; 102: 330-54

20. Abrams JA, Brar P, Diamond B, Rotterdam H, Green PH. Utility in clinical practice of immunoglobulin $A$, anti-tissue transglutaminase antibody for the diagnosis of celiac disease. Clin Gastroenterol Hepatol. 2006; 4: 726-730.

21. Donaldson MR, Firth SD, Wimpee H, Leiferman KM, Zone JJ, Horsley W, et al. Correlation of duodenal histology with tissue transglutaminase and endomysial antibody levels in pediatric celiac disease. Clin Gastroenterol Hepatol. 2007; 5: 567-573.

22. McAllister BP, Williams E, Clarke K. A comprehensive review of celiac disease/gluten-sensitive enteropathies. Clin Rev Allergy Immunol. 2019; 57: 226-243.

23. Sategna-Guidetti C, Solerio E, Scaglione N, Almo G, Mengazzi G. Duration of gluten exposure in adult coeliac disease does not correlate with the risk of autoimmune disorders. Gut. 2001; 49: 502-505.

24. Losurdo G, Principi M, lannone A, Amoruso A, lerardi E, Di Leo A, et al. Extra-intestinal manifestations of non-celiac gluten sensitivity: An expanding paradigm. World J Gastrenterol. 2018; 24: 1521-1530.

25. Mahadev S, Laszkowska M, Sundström J, Björkholm M, Lebwohl B, Green PH, et al. Prevalence of celiac disease in patients with iron deficiency anemia: A systematic review with metaanalysis. Gastroenterology. 2018; 155: 374-382.

26. Halfdanarson TR, Litrow MR, Murrayer JA. Hematologic manifestations of celiac disease. Blood. 2007; 109: 412-421.

27. Mody RJ, Brown PI, Wechsler DS. Refractory iron deficiency anemia as the primary clinical manifestation of celiac disease. J Pediatr Hematol Oncol. 2003; 25: 169-172.

28. Jatla $M$, Zemel BS, Bierly $P$, Verma $S$. Bone mineral content deficits on the spine and the whole body in children at time of diagnosis with celiac disease. J Pediatr Gastroenterol Nutr. 2009; 48: 175-180.

29. Younes M, Ben Youssef H, Safer L, Fadoza H, Zrour S, Beijal, et al. Prevalence of bone loss in adult celiac disease and associated factors: A control case study. Tun Med. 2012; 90: 129-135.

30. Karpati S. Dermatitis herpetiformis. Clin Dermatol. 2012; 30: 56-59.

31. Reunala T. Dermatitis herpetiformis: Celiac disease of the skin. Ann of Med. 1998; 30: 416-418.

32. Salmi T, Hervonen K. Current concepts of dermatitis herpetiformis. Acta Derm Venereol. 2020; 100: adv00056. 
33. Costa LM, Cappel MA, Keeling JH. Clinical, pathologic, and immunologic features of pemphigus herpetiformis: A literature review and proposed diagnostic criteria. Int J Dermatol. 2019; 58: 997-1007.

34. Schultz B, Hook K. Bullous diseases in children: A review of clinical features and treatment options. Paediatr Drugs. 2019; 21: 345-356.

35. Antiga E, Maglie R, Quintarelli L, Verdelli A, Bonciani D, Bonciolini V, et al. Dermatitis herpetiformis: Novel perspectives. Front Immunol. 2019; 10: 1290.

36. Winkler C, Jolink M, Knopff A, Kwarteng NA, Achenbach P, Bonifacio E, et al. Age, HLA, and sex define a marked risk of organ-specific autoimmunity in first-degree relatives of patients with type 1 diabetes. Diabetes Care. 2019; 42: 1684-1691.

37. Bajor J, Szakács Z, Farkas N, Hegyi P, Illés A, Solymár M, et al. Classical celiac disease is more frequent with a double dose of HLA-DQB1*02: A systematic review with meta-analysis. PLoS One. 2019; 14: e0212329.

38. Svensson J, Schwandt A, Pacaud D, Beltrand J, Birkebaek NH, Cardona-Hernandez R, et al. The Influence of treatment, age at onset, and metabolic control on height in children and adolescents with type 1 diabetes: A sweet collaborative study. Pediatr Diabetes. 2018; 1: 1441-1450.

39. Binder E, Rohrer T, Denzer C, Marg W, Ohlenschläger U, Schenk-Huber H, et al. Screening for coeliac disease in 1624 mainly asymptomatic children with type 1 diabetes: Is genotyping for coeliac-specific human leucocyte antigen the right approach? Arch Dis Child. 2019; 104: 354359.

40. Forde L, McGrath N, Devaney D, Awadalla S, McDonnell CM, Murphy NP. Coeliac screening in a high-risk population: Paediatric type 1 diabetes-a review of current guidelines and practice. Ir J Med Sci. 2019; 188: 135-139.

41. Minelli R, Gaiani F, Kayali S, Di Mario F, Fornaroli F, Leandro G, et al. Thyroid and celiac disease in pediatric age: A literature review. Acta Biomed. 2018; 89: 11-16.

42. Choung RS, Larson SA, Khaleghi S, Rubio-Tapia A, Ovsyannikova IG, King KS, et al. Prevalence and morbidity of undiagnosed celiac disease from a community-based study. Gastroenterology. 2017; 152: 830-839.

43. Gupta V, Singh A, Khadgawat R, Agarwal A, Iqbal A, Mehtab W, et al. The spectrum of clinical and subclinical endocrinopathies in treatment-naïve patients with celiac disease. Indian J Gastroenterol. 2019; 38, 518-526.

44. Fallahi P, Ferrari SM, Ruffilli I, Elia G, Biricotti M, Vita R, et al. The association of other autoimmune diseases in patients with autoimmune thyroiditis: Review of the literature and report of a large series of patients. Autoimmune Rev. 2016; 15: 1125-1128.

45. Cicarelli G, Della RG, Amboni M, Ciacci C, Mazzacca G, Filla A, et al. Clinical and neurological abnormalities in adult celiac disease. Neurol Sci. 2003; 24: 311-317.

46. Chin RL, Sander HW, Brannagan TH, Green PR, Hays AP, Alaedini A, et al. Celiac Neuropathy Neurology. 2003; 60: 1581-1585.

47. Kaplan JG, Pack D, Horoupian D, De Sousa T, Brin M, Schaumburg H. Neuropathy associated with chronic gluten enteropathy: A treatable disorder. Neurology. 1988; 38: 642-645.

48. Mearns ES, Taylor A, Thomas Craig KJ, Puglielli S, Leffler DA, Sanders DS, et al. Neurological manifestations of neuropathy and ataxia in celiac disease: A systematic review. Nutrients. 2019; 11: 380. 
49. Rodrigo L, Hernández-Lahoz C, Lauret E, Rodriguez-Peláez $M$, Soucek M, Ciccocioppo R, et al. Gluten ataxia is better classified as non-celiac gluten sensitivity than as celiac disease: $A$ comparative clinical study. Immunol Res. 2016; 64: 558-564.

50. Hadjivassiliou M, Croall ID, Zis P, Sarrigiannis PG, Sanders DS, Aeschlimann P, et al. Neurologic deficits in patients with newly diagnosed celiac disease are frequent and linked with autoimmunity to transglutaminase 6. Clin Gastroenterol Hepatol. 2019; 17: 2678-2686.

51. Dieterich $W$, Ehnis $T$, Bauer M, Donner $P$, Volta $U$, Riecken EO, et al. Identification of tissue transglutaminase as the autoantigen of celiac disease. Nat Med. 1997; 3: 797-801.

52. Sárdy M, Kárpáti S, Merkl B, Paulsson M, Smyth N. Epidermal transglutaminase (TG-ase 3 ) is the autoantigen of dermatitis herpetiformis. J Exp Med. 2002; 195: 747-757.

53. Hadjivassiliou M, Aeschlimann P, Sanders DS, Maki M, Kaukinen K, Grunewald RA, et al. Transglutaminase 6 antibodies in the diagnosis of gluten ataxia. Neurology. 2013; 80: 17401745.

54. Rouvroye MD, Zis P, Van Dam AM, Rozemuller AJ, Bouma G, Hadjivassiliou M. The neuropathology of gluten-related neurological disorders: A systematic review. Nutrients. 2020; 12: 822.

55. Vinagre-Aragón A, Zis P, Grunewald RA, Hadjivassiliou M. Movement disorders related to gluten sensitivity: A systematic review. Nutrients. 2018; 10: 1034.

56. Pratesi R, Modelli IC, Martins RC, Almeida PL, Gandolf L. Celiac disease and epilepsy: Favorable outcome in a child with difficult to control seizures. Acta Neurol Scand. 2003; 108: 290-293.

57. Gobbi G, Bouquet F, Greco L, Lambertini A, Tassinari CA, Ventura A, et al. Coeliac disease, epilepsy, and cerebral calcifications. Lancet. 1992; 340: 439-443.

58. Collin P, Pirttilä T, Nurmikko T, Somer H, Erilä T, Keyriläinen O. Celiac disease, brain atrophy, and dementia. Neurology. 1991; 41: 372-375.

59. Hu WT, Murray JA, Greenaway MC, Parisi JE, Josephs KA. Cognitive impairment and celiac disease. Arch Neurol. 2006; 63: 1440-1446.

60. Dimitrova AK, Ungaro RC, Lebwohl B, Lewis SK, Tennyson CA, Green MW, et al. Prevalence of migraine in patients with celiac disease and inflammatory bowel disease. Headache. 2013; 53: 344-355.

61. Burk K, Farecki ML, Lamprecht G, Roth G, Decker P, Weller M, et al. Neurological symptoms in patients with biopsy proven celiac disease. Mov Disord. 2009; 24: 2358-2362.

62. Lionetti E, Francavilla R, Maiuri L, Ruggieri M, Spina M, Pavone $P$, et al. Headache in pediatric patients with celiac disease and its prevalence as a diagnostic clue. J Pediatr Gastroenterol Nutr. 2009; 49: 202-207.

63. Mormile R. Celiac disease and migraine: Is there a common backstage? Int J Colorectal Dis. 2014; 29: 1571.

64. Hadjivassiliou M, Grünewald RA, Lawden M, Davies-Jones GA, Powell T, Smit CM. Headache and CNS white matter abnormalities associated with gluten sensitivity. Neurology. 2001; 56: 385-388.

65. Hadjivassiliou M, Grünewald R, Sharrack B, Sanders D, Lobo A, Williamson C, et al. Gluten ataxia in perspective: Epidemiology, genetic susceptibility and clinical characteristics. Brain. 2003; 126: 685-691.

66. Freeman HJ. Neurological disorders in adult celiac disease. Can J Gastroenterol. 2008; 22: 909911. 
67. Ameghino L, Farez MF, Wilken M, Goicochea MT. Headache in patients with celiac disease in response to the gluten-free diet. Oral Facial Pain Headache. 2019; 33: 294-300.

68. Croall ID, Hoggard N, Aziz I, Hadjivassiliou M, Sanders DS. Brain fog and non-coeliac gluten sensitivity: Proof of concept brain MRI pilot study. PLoS One. 2020; 15: e0238283.

69. Losurdo G, Principi M, lannone A, Amoruso A, lerardi E, Di Leo A, et al. Extra-intestinal manifestations of non-celiac gluten sensitivity: An expanding paradigm. World J Gastroenterol. 2018; 24: 1521-1530.

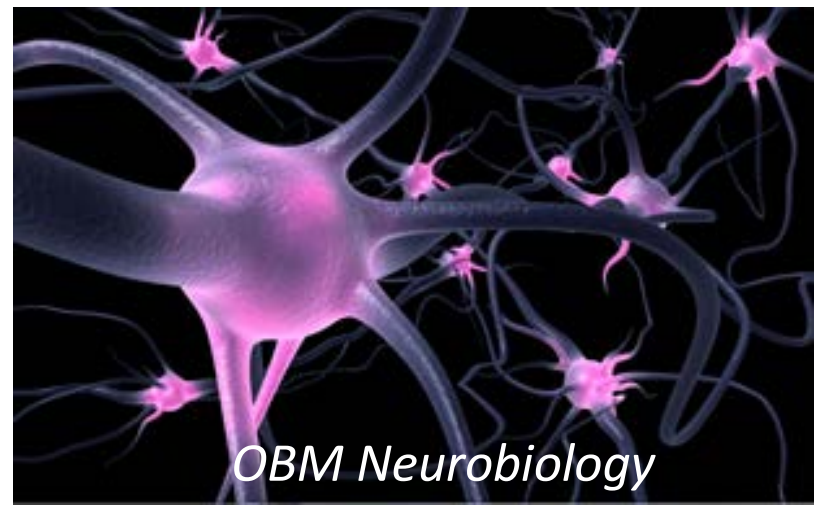

Enjoy OBM Neurobiology by:

1. Submitting a manuscript

2. Joining volunteer reviewer bank

3. Joining Editorial Board

4. Guest editing a special issue

For more details, please visit:

http://www.lidsen.com/journals/neurobiology 